University of Montana

ScholarWorks at University of Montana

6-1-1977

\title{
Light Scattering Studies of Transverse Sound Wave and Molecular Motion in Benzonitrile
}

\author{
Scott L. Whittenburg \\ University of Montana - Missoula, scott.whittenburg@umontana.edu \\ C. H. Wang \\ University of Utah
}

Follow this and additional works at: https://scholarworks.umt.edu/chem_pubs

Part of the Biochemistry Commons, Chemistry Commons, and the Physics Commons Let us know how access to this document benefits you.

\section{Recommended Citation}

Whittenburg, Scott L. and Wang, C. H., "Light Scattering Studies of Transverse Sound Wave and Molecular Motion in Benzonitrile" (1977). Chemistry and Biochemistry Faculty Publications. 67.

https://scholarworks.umt.edu/chem_pubs/67

This Article is brought to you for free and open access by the Chemistry and Biochemistry at ScholarWorks at University of Montana. It has been accepted for inclusion in Chemistry and Biochemistry Faculty Publications by an authorized administrator of ScholarWorks at University of Montana. For more information, please contact scholarworks@mso.umt.edu. 


\title{
Light scattering studies of transverse sound wave and molecular motion in benzonitrile
}

\author{
S. L. Whittenburg and C. H. Wang* \\ Department of Chemistry, University of Utah, Salt Lake City, Utah 84112 \\ (Received 19 November 1976)
}

\begin{abstract}
The zero-frequency shear wave dip appearing in the depolarized Rayleigh spectrum in benzonitrile has been studied as a function of concentration and temperature. The solution study was carried out at constant viscosity equal to the viscosity of liquid benzonitrile at each temperature. The result indicates that the presence of shear wave fine structure does not depend on the collective orientational fluctuations. The orientational and vibrational relaxation times of benzonitrile were measured at various concentrations and temperatures. The orientational relaxation times show no concentration dependence at any temperature, suggesting that the pair correlation is negligible at all concentrations. The orientational relaxation times obtained from the Raman measurements are in good agreement with the depolarized Rayleigh values at the same temperature and concentration, again indicating pair correlation is negligible in benzonitrile. Thus, both the Raman and depolarized Rayleigh scattering techniques measure the single particle relaxation time of benzonitrile. The single particle times were compared with the predictions of the hydrodynamic slip and stick models for rotational diffusion. In contrast to the results obtained for most small molecules, the stick model better approximated the experimental results.
\end{abstract}

\section{INTRODUCTION}

The depolarized Rayleigh scattering spectrum of an anisotropic liquid obtained using a double grating monochromator consists of a sharp component centered at the lase $r$ frequency superimposed upon a broad Rayleigh wing spectrum extending, in many cases, to several hundreds of wavenumbers. More precise studies of the sharp component using high resolution Fabry-Perot interferometers and single-moded lasers have shown a fine structure characterized by a doublet centered at the incident frequency. ${ }^{1,2}$ Such spectral features have been observed for many liquids ${ }^{2-11}$ and the results were initially analyzed according to a hydrodynamic theory of Rytov. ${ }^{12}$ While initial analysis of the spectra showed reasonable agreement with many features predicted by Rytov's theory, further experiments have uncovered some discrepancies. ${ }^{2}$ Several new theories based on a statistical mechanical treatment of orientational fluctuations have since been proposed, ${ }^{13-17}$ and recent experiments have shown that the statistical theories do indeed give a better description of the observed spectra. ${ }^{4-7}$ Basically, the depolarized doublet fine structure observed for the VH scattering geometry is now understood as due to scattering from shear deformation fluctuations, or in other words to scattering from nonoscillatory thermal transverse sound waves (or shear waves) These fluctuations in general are not active in light scattering; their presence in the depolarized Rayleigh spectrum is due to their coupling to the orientational fluctuations of the molecules.

Previous experimental studies of the fine structure of the depolarized Rayleigh scattering spectra have all dealt with pure liquids. The effect of dilution of the anisotropic molecules in an optically isotropic solvent on the fine spectral structure is of interest as it could yield information regarding the nature of the coupling of the transverse waves to the orientational fluctuations. In this paper, we report a detailed concentration dependent study of the depolarized Rayleigh scattering spectra of benzonitrile in an optically isotropic solvent consisting of a mixture of $\mathrm{CCl}_{4}$ and tert-butyl alcohol. The solvent was found to have negligible depolarized scattering of its own, and was prepared in such a way that it has the same viscosity at each temperature as that of liquid benzonitrile.

In addition to the study of the shear wave structure in the depolarized Rayleigh spectrum, we have also examined the orientational relaxation time as a function of temperature and a function of concentration using both the Rayleigh and Raman scattering techniques. The combination of these studies allows us to determine the pair correlation factor connecting the Rayleigh relaxation time and the single particle orientational relaxation time. ${ }^{18}$

\section{EXPERIMENTAL}

All spectra were taken with a setup similar to that used previously in this laboratory. ${ }^{19}$ The exciting source was a single frequency argon-ion laser operating at a wavelength of $488.0 \mathrm{~nm}$. Power output of the laser was about $0.6 \mathrm{~W}$ for depolarized Rayleigh scattering, and considerably less for Brillouin scattering. The scattered light was analyzed with a piezoelectrically scanned Fabry-Perot interferometer. The plate separation was varied to achieve least overlap of adjacent orders. For the polarized spectra the plate separation was $0.71 \mathrm{~cm}$ corresponding to a free spectral range of $21.0 \mathrm{GHz}$. For the depolarized spectra the plate separation was $0.47 \mathrm{~cm}$ corresponding to a free spectral range of $32.0 \mathrm{GHz}$. The finesse was typically 80 for the polarized spectra and 100 for the depolarized. The resultant signal was amplified and recorded on an $\mathrm{X}-\mathrm{Y}$ recorder.

The temperature was controlled to within $\pm 0.5 \mathrm{~K}$ with a circulating ethylene glycol-water mixture. All viscosities, densities, and indices of refraction were measured in the same constant-temperature bath.

To maintain all concentrations of benzonitrile at the viscosity of the pure liquid a $45 \%$ by volume mixture of 
$\mathrm{CCl}_{4}$ in tert-butyl alcohol was prepared as a solvent. The solutions were repeatedly filtered through $0.22 \mu \mathrm{m}$ Millipore filters into rectangular sample cells to remove dust. All liquids were spectro-quality grade.

For Raman scattering, the incident light at $488.0 \mathrm{~nm}$ from a argon-ion laser was typically $0.6 \mathrm{~W}$. The scattered light was analyzed with a Spex 1401 double grating monochromator. The resulting signal was processed by a photon counting system, and the final signal was displayed on a strip-chart recorder. The same samples as used in the Rayleigh measurements were employed in the Raman measurements.

\section{RESULTS AND DISCUSSION}

Several authors have calculated the depolarized Rayleigh scattering spectral lineshape using statistical mechanics. ${ }^{13-17}$ Keyes and Kivelson have calculated the $\mathrm{VH}$ and HH spectra (we consider in this paper only the VH spectrum). ${ }^{14}$ Their expression for the VH spectrum contains two parameters: $R$, the coupling parameter and $\Gamma$, the rate of orientational relaxation. The spectral density for $90^{\circ}$ scattering is given by

$$
\begin{aligned}
& I_{\mathrm{VH}}(\omega) \propto \frac{1}{2} \frac{\Gamma}{\omega^{2}+\Gamma^{2}} \\
& \quad+\frac{1}{2} \frac{\omega^{2} \Gamma+\Gamma\left(q^{2} \eta / \rho\right)^{2}(1-R)}{\left(\Gamma q^{2} \eta / \rho-\omega^{2}\right)^{2}+\omega^{2}\left[\Gamma+(1-R)\left(q^{2} \eta / p\right)\right]^{2}},
\end{aligned}
$$

where $\eta$ is the viscosity, $\rho$ is the density, and $q$ is the amplitude of the scattering vector given by

$$
q=\left(2 n / \lambda_{0}\right) \sin (\theta / 2),
$$

where $n$ is the index of refraction, $\lambda_{0}$ the wavelength of the incident light in vacuum, and $\theta$ the scattering angle.

Similar expressions of Eq. (1) were also obtained by Andersen and Pecora, ${ }^{15}$ and by Gershon and Oppenheim. ${ }^{17}$ However, the expression for the coupling parameter $R$ in the derivation of Keyes and Kivelson was in error as it depends only on the static correlation factor which can be shown to vanish identically. 4,17 The expression for $R$ is nonvanishing in Refs. 15 and 17 .

In $\mathrm{Eq}$. (1), $\Gamma$ is the half-width at half-height of the orientational spectrum and is related to the Rayleigh relaxation time, $\tau_{\text {Ray }}$, by

$$
\tau_{\mathrm{R} \text { m }}=(2 \pi \Gamma)^{-1} \text {. }
$$

The coupling parameter $R$ is defined by, ${ }^{15}$

$$
R=1-\Gamma^{\prime} \rho / q^{2} \eta
$$

where $\Gamma^{\prime}$ is the relaxation rate of the shear wave.

The Rayleigh relaxation time $\tau_{\mathrm{Ray}}$ is related to the single particle orientational relaxation time $\tau$, by $^{18}$

$$
\tau_{\mathrm{Ray}}=F \tau_{s}=(1+f n / 1+g n) \tau_{s},
$$

where the pair correlation factor $F$ depends on the static pair correlation $f$, the dynamic pair correlation $g$, and the number density $n$ of the scatterer. As benzonitrile is diluted with an optically isotropic solvent at the same viscosity as that of pure benzonitrile, the coupling factor $R$ and the pair correlation factor $F$ are expected to

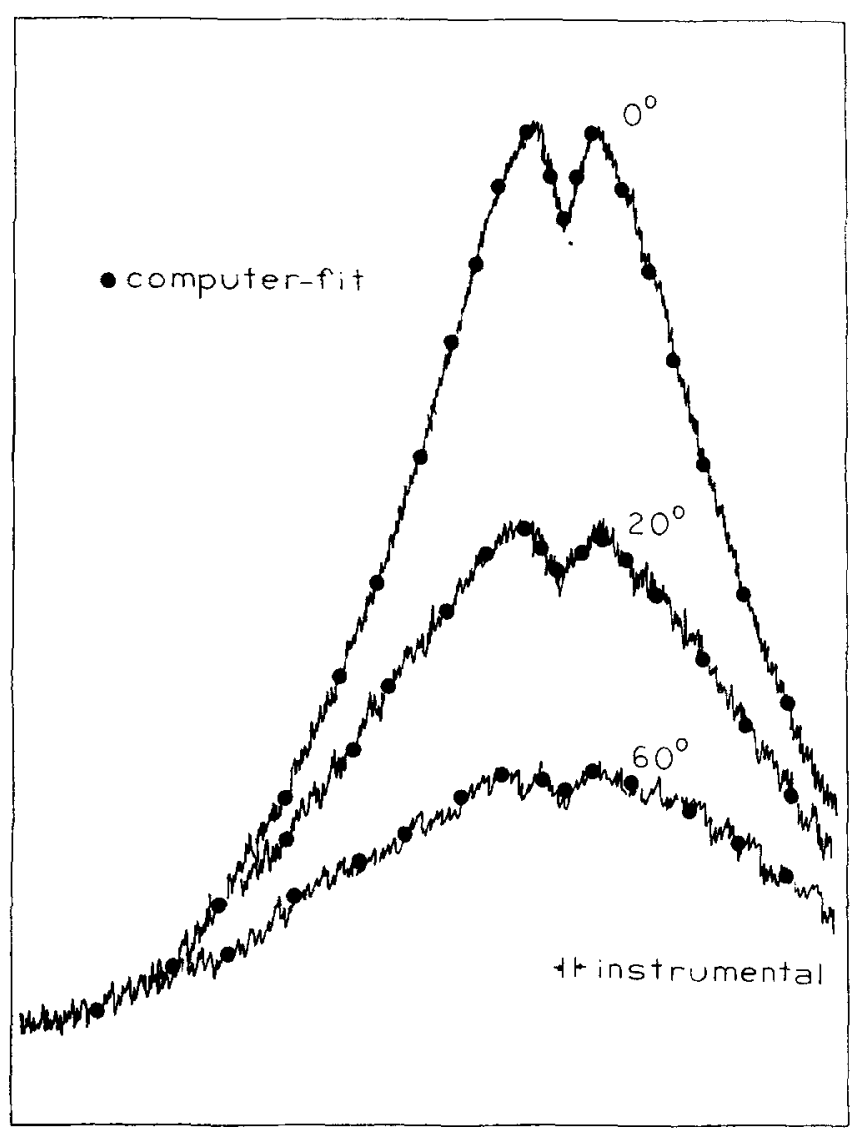

FIG. 1. The experimental and theoretically calculated spectra of pure benzonitrile at three different temperatures.

change, thus resulting in a change of the doublet structure in the sharp portion of the depolarized Rayleigh spectrum. In the absence of the shear wave phenomena, the single particle relaxation time, $\tau_{s}$, can be determined from the concentration dependence of $\tau_{\mathrm{Ray}}{ }^{19}$ The importance of the pair correlation factor can then be determined using Eq. (5).

We have measured depolarized spectra of pure benzonitrile at eight different temperatures in the range between $263-353 \mathrm{~K}$. The depolarized spectra at $83 \%$, $70 \%$, and $50 \%$ concentrations at various temperatures were also recorded. All spectra display over the temperature and concentration ranges a doublet structure. The width of the doublet changes with temperature and concentration. The experimental spectra were fitted numerically to Eq. (1) with the aid of a nonlinear least squares fitting program, using the measured values of $q, \eta$, and $\rho$ and treating $\Gamma$ and $R$ as parameters. Typical fits are shown in Fig. 1. Appropriate numerical procedures were used to correct the instrumental function and overlap of adjacent orders. The resulting values of the coupling parameter $R$ and the Rayleigh relaxation time $\tau_{\text {Ray }}\left[=(2 \pi \Gamma)^{-1}\right]$ are given respectively in Tables I and II, along with the $\eta / T$ values.

\section{A. Transverse sound wave}

The values deduced for $R$, the coupling parameter, are shown in Fig. 2 as a function of temperature for solutions of various concentrations. One notes that, 
TABLE I. $R$ and the shear wave relaxation time $\tau_{\mathbf{s w}}$ as a function of concentration, temperature, and viscosity.

\begin{tabular}{|c|c|c|c|c|c|c|c|c|c|c|c|c|}
\hline \multirow[b]{3}{*}{$T(\mathrm{~K})$} & \multirow[b]{3}{*}{$R$} & \multirow{3}{*}{$\begin{array}{r}100 \% \\
\tau_{s w}(\mathrm{ps})\end{array}$} & \multirow[b]{3}{*}{$\eta^{\mathrm{a}} / T \times 10^{3}$} & \multirow[b]{3}{*}{$R$} & \multirow{3}{*}{$\begin{array}{r}83 \% \\
\tau_{\mathrm{sw}}(\mathrm{ps})\end{array}$} & \multirow{2}{*}{\multicolumn{2}{|c|}{ Concentration }} & \multirow{2}{*}{\multicolumn{2}{|c|}{$70 \%$}} & \multirow{2}{*}{\multicolumn{3}{|c|}{$50 \%$}} \\
\hline & & & & & & & & & & & & \\
\hline & & & & & & $\eta / T \times 10^{3}$ & $R$ & $\tau_{\mathrm{sw}}(\mathrm{ps})$ & $\eta / T \times 10^{3}$ & $R$ & $\tau_{\mathrm{sw}}(\mathrm{ps})$ & $\eta / T \times 10^{3}$ \\
\hline 263 & 0.40 & 151 & 8.77 & & & & & & & & & \\
\hline 273 & 0.34 & 165 & 6.98 & 0.36 & 1.12 & 6.98 & 0.27 & 1.17 & 7.03 & 0.27 & 183 & 6.89 \\
\hline 283 & 0.19 & 161 & 5.62 & 0.20 & 1.07 & 5.62 & 0.20 & 1.25 & 5.69 & 0.12 & 180 & 5.44 \\
\hline 293 & 0.16 & 183 & 4.56 & 0.22 & 1.30 & 4.56 & 0.18 & 1.44 & 4.64 & & & \\
\hline 303 & 0.11 & 200 & 3.38 & 0.20 & 1.48 & 3.83 & 0.12 & 1.58 & 3.84 & & & \\
\hline 313 & 0.14 & 238 & 3.19 & 0.14 & 1.59 & 3.19 & 0.14 & 1.86 & 3.20 & & & \\
\hline 333 & 0.12 & 302 & 2.30 & 0.10 & 1.97 & 2.30 & & & & & & \\
\hline 353 & 0.05 & 351 & 1.59 & & & & & & & & & \\
\hline
\end{tabular}

ânits of $\mathrm{cp}-\mathrm{K}^{-1}$.

over the temperature and concentration ranges, $R$ is much smaller than unity; furthermore it decreases with increasing temperature. This result is consistent with the result recently obtained in liquid salol, but is in contrast to the results for anisaldehyde, ${ }^{4}$ acetophenone, ${ }^{8}$ and triphenyl phospite, ${ }^{7}$ in which systems the coupling parameters are found to be insensitive to temperature.

The temperature dependence of the shear wave relaxation rate $\Gamma^{\prime}$ can be obtained from the coupling parameter $R$. We have measured $\rho, q$, and $\eta$ for all solutions as a function of temperature, and have used these values to determine $\Gamma^{\prime}$ using Eq. (4) and $R$. The transverse sound (or shear wave) relaxation time $\tau_{s w}$ is defined as $\tau_{s w}=\left(2 \pi \Gamma^{\prime}\right)^{-1}$. The values of $\tau_{s w}$ are shown in Fig. 3 as a function of $\eta / T$ for various concentrations. One notes that, except for the $50 \%$ solution which does not follow the same pattern, at a given $\eta / T$ value the shear wave relaxation times for other solutions increase with increasing dilution. Moreover, the shear relaxation time depends on $\eta / T$ nonlinearly and decreases with increasing $\eta / T$, approaching a plateau value for large $\eta / T$. This concentration and temperature dependence is quite different from the behavior of the single particle reorientational relaxation time $\tau_{s}$ (as shown in Fig. 4) whose value has been shown to increase with increasing $\eta / T$ and depends only on the $\eta / T$ value, irrespective of concentration. In the present work, we maintained the solution to have the same viscosity as that of liquid benzonitrile at the same temperature, the change of the shear relaxation time with concentration is not due to the viscosity effect, but is due to the change of liquid structure which has caused the vibrational relaxation time to vary. We do not have at present a molecular theory which can account for the observed temperature and concentration dependencies of the shear wave relaxation time. It should be mentioned that the temperature dependence of $\tau_{s w}$ we have observed in liquid benzonitrile is similar to that of liquid salol ${ }^{11}$ in the $q^{2} \eta / \rho \Gamma<1$ region.

\section{B. Molecular reorientation}

In Fig. $4 \tau_{\text {Ray }}$ values for $100 \%, 83 \%, 70 \%$, and $50 \%$ solutions are plotted as a function of $\eta / T$. One data point obtained previously by Stegeman and Stoicheff for the pure liquid at room temperature was also included in Fig. 4 for comparison. The agreement is excellent.
The $\tau_{\text {Ray }}$ values for various concentrations between 263 and $333 \mathrm{~K}$ are also given in Table II.

A linear least squares fit of the $\tau_{\mathrm{Ra}}$ values to the equation,

$$
\tau_{\text {Ray }}=C(\eta / T)+\tau_{0}
$$

gives $C=(4.3 \pm 0.25) \times 10^{3} \mathrm{ps} \mathrm{K} / \mathrm{cp}$ and $\tau_{0}=(0.0 \pm 0.05)$ ps. All solution data follow the same linear curve, thus indicating that the pair correlation factor $F$ is independent of concentration, and thus must be equal to unity. According to Eq. (5) the pair correlation factor depends on both the static correlation $f$ and the dynamic correlation $g$ and concentration $n$. The fact that $F$ is equal to unity at all concentrations suggest that $f=g=0$ for benzonitrile, and $\tau_{\text {Ray }}$ is equivalent to the single particle orientational correlation time.

We found previously a similar result in liquid acetonitrile. ${ }^{20}$ The result is quite puzzling as one would generally anticipate the pair correlation factor to be determined in part by the magnitude of the permanent dipole moment of the molecule. Due to the fact that benzonitrile has a fairly large dipole moment, one would expect to find the pair correlation factor $F$ to be greater than 1 and also concentration dependent. The contrary was found both in acetonitrile and benzonitrile, and this indicates that the premanent dipole moment plays no role in determining the pair correlation factor. There is at present little understanding as to what molecular parameters are responsible for the pair correla-

TABLE II. Rayleigh relaxation times.

\begin{tabular}{llllll}
\hline \hline & & $\tau_{\text {Ras }}$ & & & \\
$T(\mathrm{~K})$ & $100 \%$ & $83 \%$ & $70 \%$ & $50^{\mathrm{a} \%}$ & $\eta / T \times 10^{3}(\mathrm{cp} / \mathrm{K})$ \\
\hline 263 & 36.7 & & & & 8.77 \\
273 & 32.4 & 32.0 & 32.6 & 33.5 & 6.98 \\
283 & 25.0 & 25.0 & 25.2 & 26.1 & 5.62 \\
293 & $21.6^{\text {b }}$ & 21.5 & 21.0 & 20.9 & 4.57 \\
303 & 18.2 & 18.0 & 17.9 & 18.2 & 3.83 \\
313 & 15.3 & 15.4 & 15.0 & 14.4 & 3.17 \\
323 & & & & 12.4 & 2.67 \\
333 & 11.6 & & & & 2.30 \\
\hline \hline
\end{tabular}

${ }^{2}$ Minimal viscosity scaling required to equate to viscosity of pure benzonitrile.

Other studies, Ref. $3,19.4$ ps (295.5 K). 


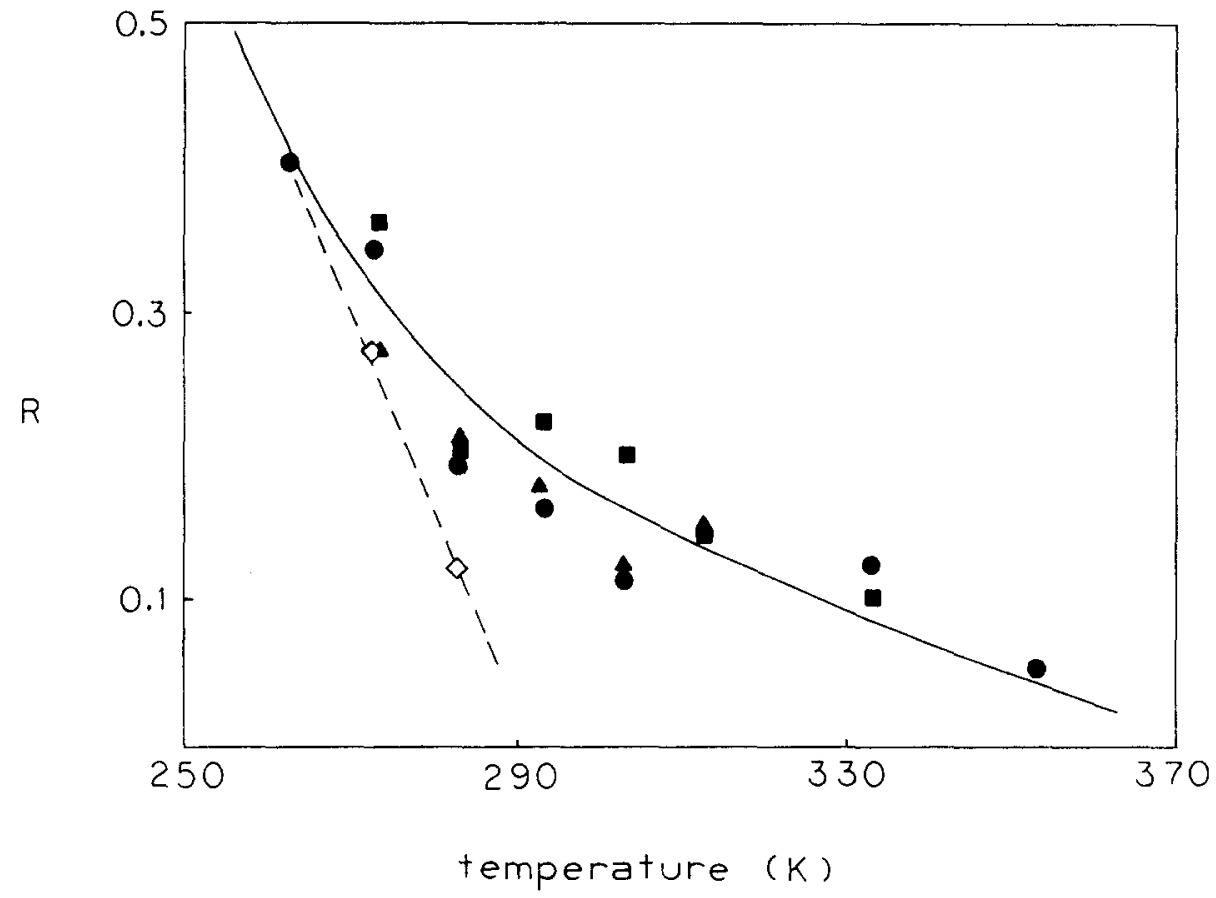

FIG. 2. The coupling parameter, $R$, as a function of temperature and concentration. $\bullet$, $\square, \Delta, \bigcirc$ are from depolarized Ralyeigh measurements on $100 \%, 83 \%, 70 \%, 50 \%$ by volume solutions, respectively.

tion factor $F$. More experimental and theoretical studies to clarify this problem are needed.

If a rate-activated process is assumed for the temperature dependence of the molecular reorientation relaxation times, the values of $\tau_{\text {Ray }}$ lead to an activation energy of $3.05 \mathrm{kcal} / \mathrm{mole}$. This activation energy is mainly due to the activation energy in viscous flow (derived from the viscosity), and is fairly comparable with values obtained in liquids consisting of weakly interacting molecules.

The fact that $\tau_{\text {Ray }}$ shows negligible concentration dependence indicates that it is equivalent to the single particle reorientation time. This result is corroborated by the Raman scattering study of the $\mathrm{C} \equiv \mathrm{N}$ stretching mode in benzonitrile. Raman scattering is generally an incoherent process and the spectral density of a Raman band is determined mainly by the single particle cor relation function. When certain approximations are valid, it is possible to determine the single particle reorientation time from the polarized and depolarized Raman spectra of a totally symmetric vibration. Cheung, etal. have discussed these assumptions and their applicability. ${ }^{19}$ We have measured the Raman relaxation times, $\tau_{\text {ram }}$ and $\tau_{\text {iso, }}$, corresponding respectively to molecular reorientation and vibrational relaxations. ${ }^{20}$ The results are shown in Table III. The $\tau_{\text {Ram }}$ data as a function of $\eta / T$ are shown in Fig. 4. They

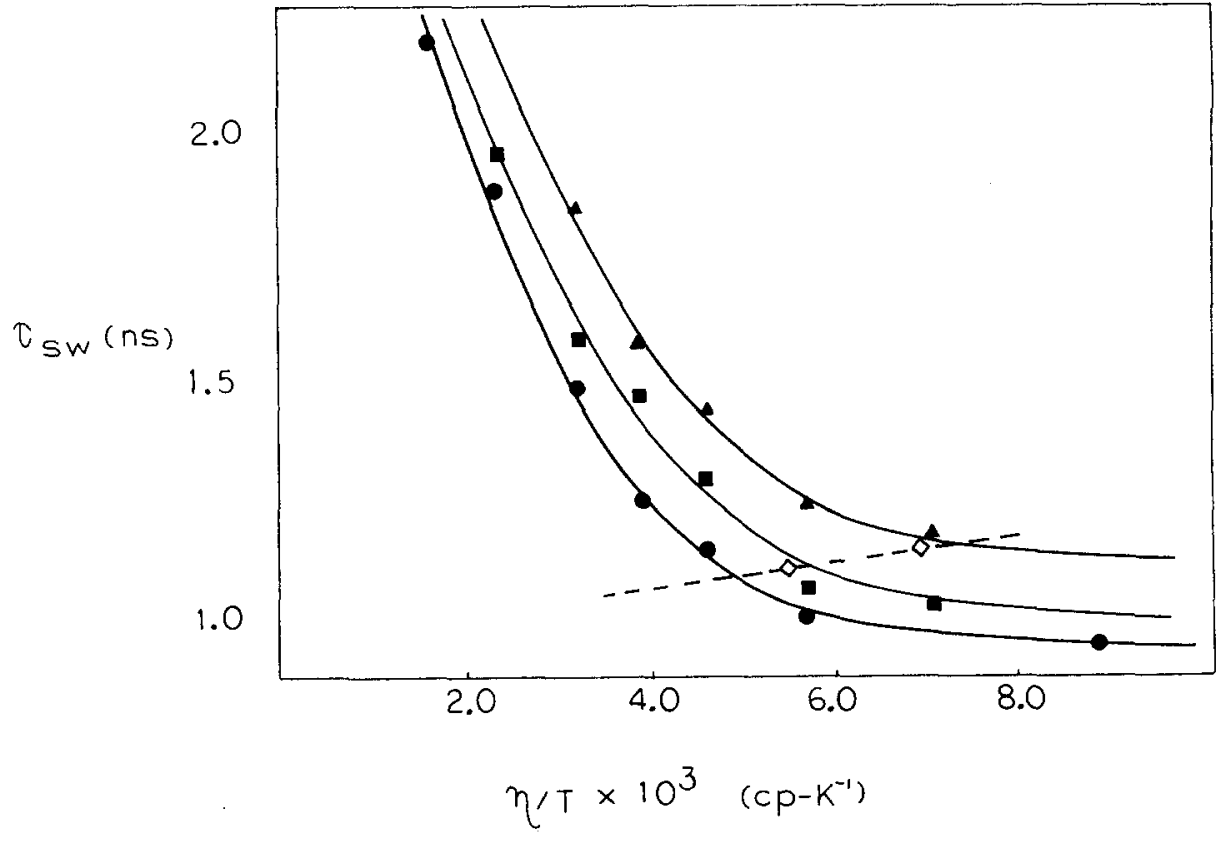

FIG. 3. The shear wave relaxation time, $\tau_{\mathbf{s w}}$, as a function of viscosity, temperature, and concentration. $\bullet, \bullet, \wedge, 0$ correspond to solutions of $100 \%, 83 \%, 70 \%, 50 \%$ by volumes mixtures of benzonitile in a constant viscosity solvent. 


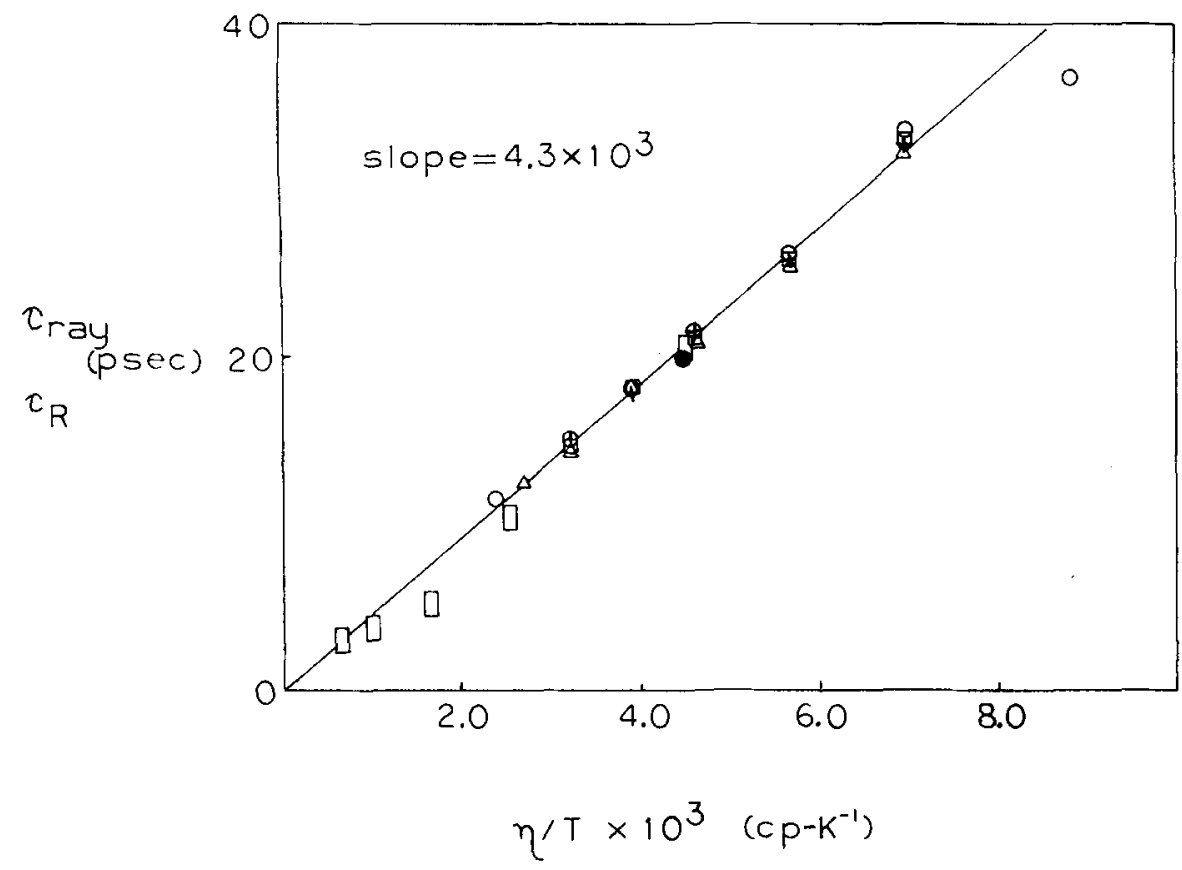

FIG. 4. Single particle relaxation time as function of $\eta / T$. $\square$ is from Raman measurement; $0, \Delta, \quad \square,+$ are from depolarized Rayleigh measurements on $100 \%, 83 \%, 70 \%, 50 \%$ by volume solutions respectively; $\bullet$ is from Ref. 3 .

follow closely the $\tau_{\text {Ray }}$ results, indicating that $\tau_{\text {Ram }}$ as sociated with the $\mathrm{C} \equiv \mathrm{N}$ stretching vibration is a good measure of the single particle molecular reorientation time, and the type of pair correlations as defined in light scattering are not likely to be important.

This result indicates therefore that the presence of shear wave fine structure does not depend on the collective orientational fluctuations, as has generally been assumed in the various statistical mechanical theories of the depolarized Rayleigh scattering spectra. ${ }^{13-17}$ The shear wave can couple to single particle reorientational motion by which the shear stress is relieved; no pair orientational correlated motion is necessary, at least not in benzonitrile.

Having obtained the single particle reorientational relaxation times as a function of $\eta / T$, we can compare our data with what is predicted by the hydrodynamic models associated with the Stokes-Einstein relation. If the stick boundary condition is assumed, the StokesEinstein relation for the single particle relaxation time is

$$
\tau_{s}=(V / k) \eta / T
$$

where $V$ is the molecular volume. Using the prescription of Edward to calculate the molecular volume, ${ }^{21}$ we

TABLE III. Raman relaxation times.

\begin{tabular}{llllll}
\hline \hline$(\mathrm{K})$ & $\tau_{\text {iso }}{ }^{2}(\mathrm{ps})$ & $\tau_{R}{ }^{\mathrm{a}}(\mathrm{ps})$ & $\eta / T \times 10^{3}(\mathrm{cp} / \mathrm{K})$ & Volume\% & $\tau_{\text {iso }}{ }^{\mathrm{b}}$ \\
\hline 295 & 1.95 & 20.4 & 4.41 & 100 & 1.93 \\
313 & 1.84 & 13.3 & 3.19 & 83 & 1.84 \\
333 & 1.75 & 5.2 & 2.30 & 70 & 1.80 \\
353 & 1.69 & 3.9 & 1.67 & 50 & 1.62 \\
373 & 1.62 & 3.4 & 1.27 & & \\
\hline \hline
\end{tabular}

apure benzonitrile.

$b_{297} \mathrm{~K}$ viscosity equal to that of pure benzonitrile. have calculated the $V / k$ values modeling benzonitrile as a sphere and as an ellipsoid. The calculated results are given in Table IV, along with the experimental result. The calculation of the Stokes-Einstein slope for an ellipsoid with slip boundary conditions ${ }^{22}$ is also given in Table IV. Although there is ambiguity regarding the calculation of the molecular volume of benzonitrile, the calculated results are in reasonable agreement with the experiment. The stick model seems to agree better with the experiment, in contrast to the results of other small molecular systems ${ }^{19,20,23}$ previously investigated in this laboratory.

Better agreement with the stick model has also been found in nitrobenzene. ${ }^{24-26}$ It appears that the depolarized scattering intensity in benzonitrile is more likely due to reorientation about the axis in the ring (and perpendicular to the $\mathrm{C} \equiv \mathrm{N}$ bond) as it has been shown that reorientation about this axis follows better the stick model while reorientation about the other two axes show slip behavior. ${ }^{26}$

\section{SUMMARY AND CONCLUSIONS}

We have studied the shear wave dip in benzonitrile as a function of concentration and temperature. The ex-

TABLE IV. Comparison of theoretical Stokes-Einstein slope to experimental slope.

\begin{tabular}{|c|c|c|}
\hline $\begin{array}{l}\text { Stokes-Einstein slope for a sphere } \\
\text { with stick boundary conditions }\end{array}$ & $5.8 \times 10^{3}$ & $\frac{\mathrm{ps} \mathrm{K}}{\mathrm{cp}}$ \\
\hline $\begin{array}{l}\text { Stokes-Einstein slope for an ellipsoid } \\
\text { with stick boundary conditions }\end{array}$ & $6.0 \times 10^{3}$ & $\frac{\mathrm{psK}}{\mathrm{cp}}$ \\
\hline $\begin{array}{l}\text { Stokes-Einstein slope for an ellipsoid } \\
\text { with slip boundary conditions }\end{array}$ & $1.4 \times 10^{3}$ & $\frac{\mathrm{psK}}{\mathrm{cp}}$ \\
\hline Experimental slope & $4.3 \times 10^{3}$ & $\frac{\mathrm{psK}}{\mathrm{cp}}$ \\
\hline
\end{tabular}


perimental data was fit to the theoretical equation based on statistical mechanics. The behavior of the coupling parameter, $R$, was found to be similar to the behavior of liquid salol, yet quite different from the results of other studies of the shear wave phenomena. $R$ was found to decrease with increasing temperature. The shear wave relaxation time, $\tau_{s w}$, was found to have a markedly different viscosity and temperature dependence than $\tau_{s}$.

We have found that consistent single particle orientational relaxation times for benzonitrile are obtained by both the Raman and depolarized Rayleigh scattering techniques. The experimental data is consistent over the entire range of concentrations and temperatures studied. The depolarized Rayleigh measurements found $\tau_{\text {Ray }}$ to be independent of the concentration of scatterers. This coupled with the agreement with $\tau_{\text {Ram }}$, suggested that for benzonitrile the dynamic and static pair correlations are both zero. The experimental values for $\tau_{\text {Rer }}$ were found to be consistent with previous studies. The single particle orientational relaxation times were found to approximate the prediction of the stick hydrody namic model, in contrast to the results of the studies of most small molecules.

\section{ACKNOWLEDGEMENT}

We acknowledge the support of the Donors of the $\mathrm{Pe}$ troleum Research Fund, administered by the American Chemical Society, for support of this research.

${ }^{1}$ V. S. Starunov, E. V. Tiganov, and I. L. Fabelinski, JETP Lett. 5, 260 (1967).

${ }^{2}$ G. I. A. Stegeman and E. P. Stoicheff, Phys. Rev. Lett. 21, 202 (1968); Phys. Rev. A 7, 1160 (1973).

${ }^{3}$ G. D. Enright, G. I. A. Stegeman, and B. P. Stoicheff, J.
Phys. Radium 33, 207 (1972).

${ }^{4}$ G. R. Alms, D. R. Bauer, J. I. Brauman, and R. Pecora, J. Chem. Phys. 59, 5304 (1973).

${ }^{5} \mathrm{y}$. A. Bucaro, H. D. Hardy and R. F. Horton, J. Chem. Phys. 60, 1671 (1974).

${ }^{6}$ G. D. Enright and B. P. Stoicheff, J. Chem. Phys. 60, 2536 (1974).

${ }^{7}$ S. -J. Tsay and D. Kivelson, Mol. Phys. 29, 1 (1975).

${ }^{8}$ P. Bezot, G. M. Searby, and P. Sixou, J. Chem. Phys. 62, 3813 (1975).

${ }^{9}$ G. M. Searby, P. Bezot, and P. Sixou, Phys. Lett. A 51 , 159 (1975); J. Chem. Phys. 64, 1485 (1976).

${ }^{10}$ P. Sixou, P. Bezot, and G. M. Searby, Mol. Phys. 30, 1149 (1975).

${ }^{11}$ G. D. Enright and B. P. Stoicheff, J. Chem. Phys. 64, 3658 (1976).

${ }^{12}$ S. M. Rytov, Sov. Phys. JETP 6, 401 (1958).

${ }^{13}$ A. Ben-Reuven and N. D. Gershon, J. Chem. Phys. 54, 1049 (1971).

${ }^{14}$ T. Keyes and D. Kivelson, J. Chem. Phys. 54, 1786 (1971); 56, 1876 (1972); J. Phys. Radium 33, 231 (1972).

${ }^{15} \mathrm{H}$. C. Andersen and R. Pecora, J. Chem. Phys. 54, 2584 (1971); 55, 1496 (1971).

${ }^{16} \mathrm{~N}$. K. Ailawadi, B. J. Berne, and D. Forster, Phys. Rev. A 3, 1472 (1971).

${ }^{17}$ N. D. Gershon and I. Oppenheim, Physica (Utrecht) 64, 247 (1973); J. Chem. Phys. 59, 1337 (1973).

${ }^{18} \mathrm{~T}$. Keyes and D. Kivelson, J. Chem. Phys. 56, 1057 (1972).

${ }^{19} \mathrm{C} . \mathrm{K}$. Cheung, D. R. Jones, and C. H. Wang, J. Chem. Phys. 64, $3567(1976)$.

${ }^{20}$ S. L. Whittenburg and C. H. Wang, J. Chem. Phys, (to be published).

${ }^{21}$ J. T. Edward, J. Chem. Ed. 47, 261 (1970).

${ }^{22} \mathrm{C}$. Hu and R. Zwanzig, J. Chem. Phys. 60, 4345 (1974).

${ }^{23}$ C. H. Wang, D. R. Jones, and D. H. Christensen, J. Chem. Phys. 64, 2820 (1976).

${ }^{24}$ G. R. Alms, D. R. Bauer, J. I. Brauman, and R. Pecora, J. Chem. Phys. 59, 5310 (1973).

${ }^{25}$ D. R. Bauer, J. I. Brauman, and R. Pecora, J. Am. Chem. Soc. 96, 6840 (1974).

${ }^{26}$ B. J. Berne and R. Pecora, Dynamic Light Scattering (Wiley, New York, 1976), p. 150. 